\title{
Implementation of Virtual Medical Devices in Internet and Wireless Cellular Networks
}

\author{
Željko Obrenović \\ University of Belgrade, Belgrade, Yugoslavia \\ obren@computer.org \\ Dušan Starčević \\ University of Belgrade, Belgrade, Yugoslavia
}

Emil Jovanov
University of Alabama, Huntsvile, U.S.A

Vlada Radivojević

Polyclinic Median, Belgrade, Yugoslavia

\begin{abstract}
Telemedical systems work in heterogeneous processing environment and require a flexible system reconfiguration. Physician may switch from a personal digital assistant to a high performance - high resolution workstation very often, expecting the telemedical system to reconfigure and provide the support within seconds. In this paper we present a telemedical virtual collaborative environment based on Internet and wireless cellular network technologies. We apply a novel concept of interactive Virtual Medical Devices (VMD) and merge remote data acquisition, distributed processing, and data presentation. Using VMD we have integrated spatially distributed functions: $\mathrm{I} / \mathrm{O}$ at patient side, data processing, and presentation at physician side. Data acquisition is performed by remote medical device or medical sensor. Data processing is distributed and involves sophisticated real-time signal processing algorithms. We developed three VMD interfaces for data presentation and data distribution: WWW HTML interface, WAP WML interface, and GSM SMS interface. We have applied VMDs to facilitate distributed analysis of electroencephalogram (EEG) data in Internet environment.
\end{abstract}

Key words: Internet, Telemedicine, WAP, SMS

The original version of this chapter was revised: The copyright line was incorrect. This has been corrected. The Erratum to this chapter is available at DOI: 10.1007/978-0-387-35616-7_23 


\section{INTRODUCTION}

In conventional metaphor of health care services, physical presence and collaboration of physicians, medical staff, and patient was prerequisite. Current health care trend is to provide the latest available information technology to all segments of urban and rural society. Moreover, globalization and higher people mobility (business, tourism, etc.), leads to fragmented care delivered at scattered locations. As a consequence, usual limitations include:

- Lack of patient medical record (PMR);

- Absence of qualified physician;

- Required specialist team;

- Lack of sophisticated medical equipment.

Increased performance of information infrastructure facilitates real-time execution of applications, establishing a basis for a new medical discipline "telemedicine" [1]. In addition to improved healthcare by telepresence, telemedicine can create a new quality by creating virtual collaborative environments, independent of the participants' physical presence.

In this paper we present a telemedical virtual collaborative environment based on Internet and wireless cellular network technologies. We have applied a novel concept of interactive virtual medical devices (VMD) and merged remote data acquisition, distributed processing, and data presentation. VMDs integrate spatially distributed functions: $\mathrm{I} / \mathrm{O}$ at patient side, data processing, and presentation at physician side. This provides a new niche for specialized application service providers (ASP) that could provide VMDs and support for extended services.

Second section reviews telemedical applications based on the Internet and wireless cellular network technologies. In the third section we present framework for virtual medical device applications based on software components implementing GSM SMS, WAP WML, and WWW HTML interfaces. Section four provides implementation details and presents lessons learned. The last section concludes the paper and outlines future work.

\section{TELEMEDICINE, INTERNET, AND WIRELESS NETWORKS}

Telemedicine significantly changes storage, retrieval, and usage of biomedical information in medical information systems. Evolution of medical information systems is greatly influenced by capabilities and price/performance ratio of novel information technologies. The Internet as a global information infrastructure with World Wide Web (WWW) services, 
and wireless cellular networks providing WAP based services, offer low cost solution for telemedical applications [2, 3]. It seems that at the present state of technology, Web and WAP based medical applications present natural way of creating interactive collaborative environments - Virtual Medical Worlds as shown in Figure 1.

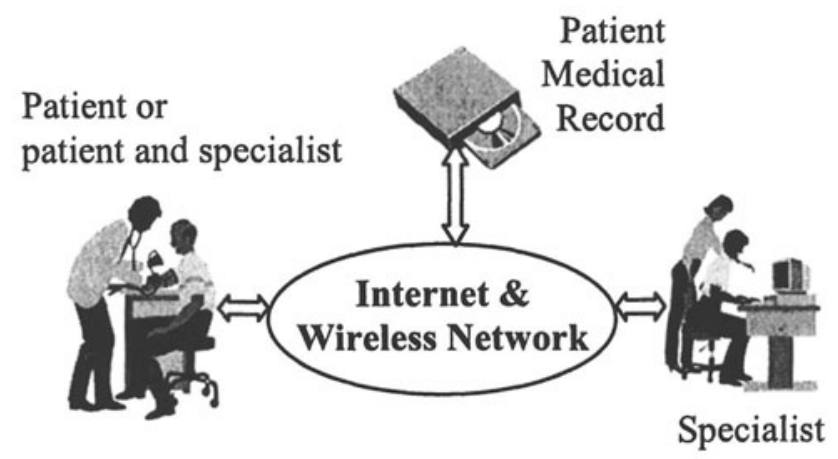

Figure 1. Virtual Medical World based on Internet and Wireless Networks technologies. Participants could be on different physical locations.

Telemedicine creates virtual environment for collaboration between multiple physicians, medical staff and the patient, independently of their physical presence [4]. Therefore, high-quality medical services became available for distant patients, remote monitoring and urgent cases. A typical example of early telemedical environment of electroencephalographic (EEG) monitoring and analysis is teleEE $G_{2}$ developed at the University of Calabria. The system gives the possibility to manage local patient database, including EEG data records. It is based on point-to-point communication using a standard telephone line [5].

The Internet and Web based medical information systems initiate and maintain ad hoc virtual medical worlds on participant's request. They provide increased flexibility of data acquisition and analysis, and allow creation of sophisticated application $[6,7]$.

Today, users increasingly adopt Internet-enabled cellular phones and other hand-held devices. WAP is platform independent wireless technologies, which help these devices effectively access Internet content, and services, and communicate with each other [8]. Therefore, WAP has great potential in telemedical application.

WAP and the Internet can support new classes of telemedical applications, such as remote monitoring using wireless personal monitors and cellular phone link established on request in the case of medical emergencies [9, 10]. Extended infrastructure for these new telemedical applications is shown in Figure 2. Telemedical applications use existing 
medical information systems with patient medical records (PMR), providing data for patients and/or physicians over the Internet or cellular phone network.

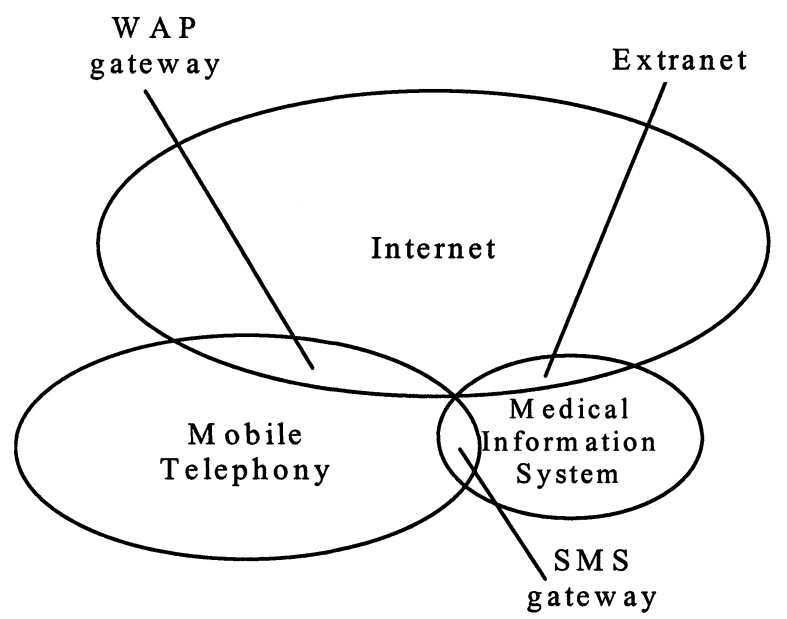

Figure 2. Infrastructure of a Virtual Medical World based on Internet and wireless networks technologies.

\section{FRAMEWORK FOR VMD APPLICATIONS}

Diagnostic procedures often require different views on the same data set or medical recording. Therefore physicians should be able to choose appropriate view or easy change different views during analysis. For example, during EEG analysis electroencephalographer can choose standard waveform view to analyze temporal changes [7]. In addition, animated 2-D or 3-D topographic maps could be used to trace spatio-temporal changes of brain electrical activity [11]. Although based on the same EEG data set, they provide the flexibility of different views within the same diagnostic procedure.

Instead of using more medical devices we can apply multiple Virtual Medical Devices (VMDs) on the same computing platform, such as PC or handheld PDA device. VMD is able to integrate spatially distributed functions: $\mathrm{I} / \mathrm{O}$ at patient side, data processing, and presentation at physician side, using Internet and mobile telephone technologies and services. Conceptual views on a VMD application framework are shown in Figure 3a. 

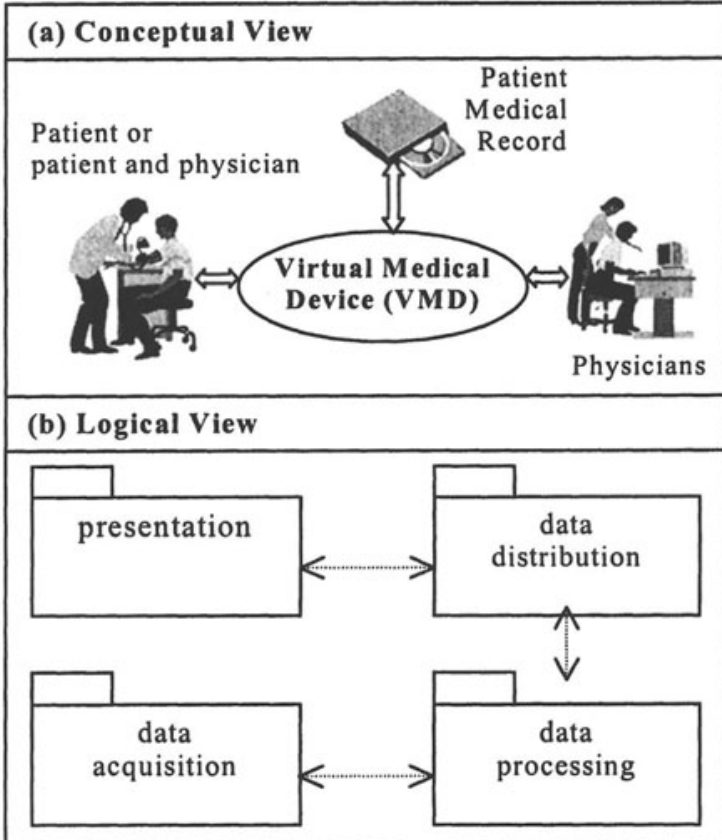

(c) Deployment View

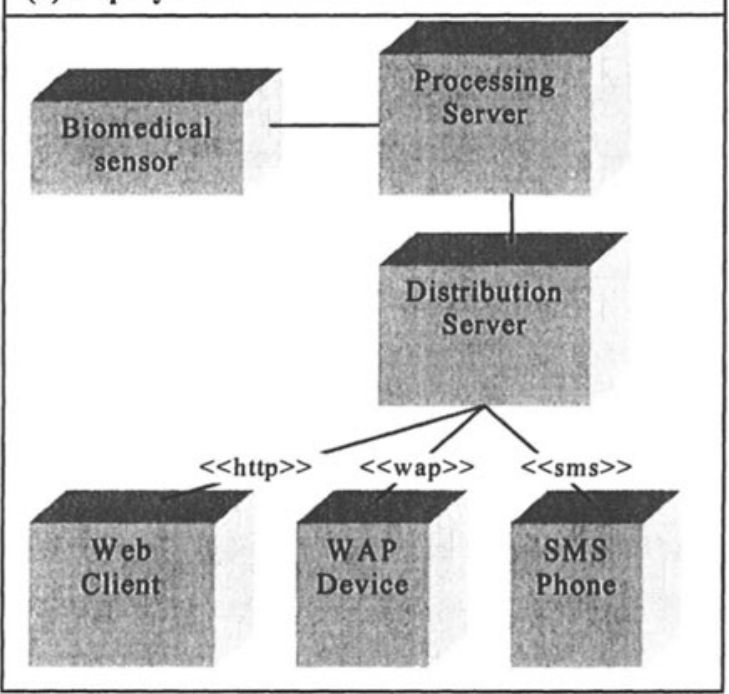

Figure 3. Different views on the proposed VMD application framework. 
In logical view shown in Figure 3b, we identify four types of components as elements of virtual medical devices: data acquisition, data distribution, data processing, and data presentation. The deployment view of a VMD application is shown in Figure 3c.

Figure 4 shows components and processes in our real-time EEG data monitoring and analysis environment. Please note that in our framework, patient can be not only an object of data acquisition, but also a subject for data presentation and control. A separate block represents each of the software components.

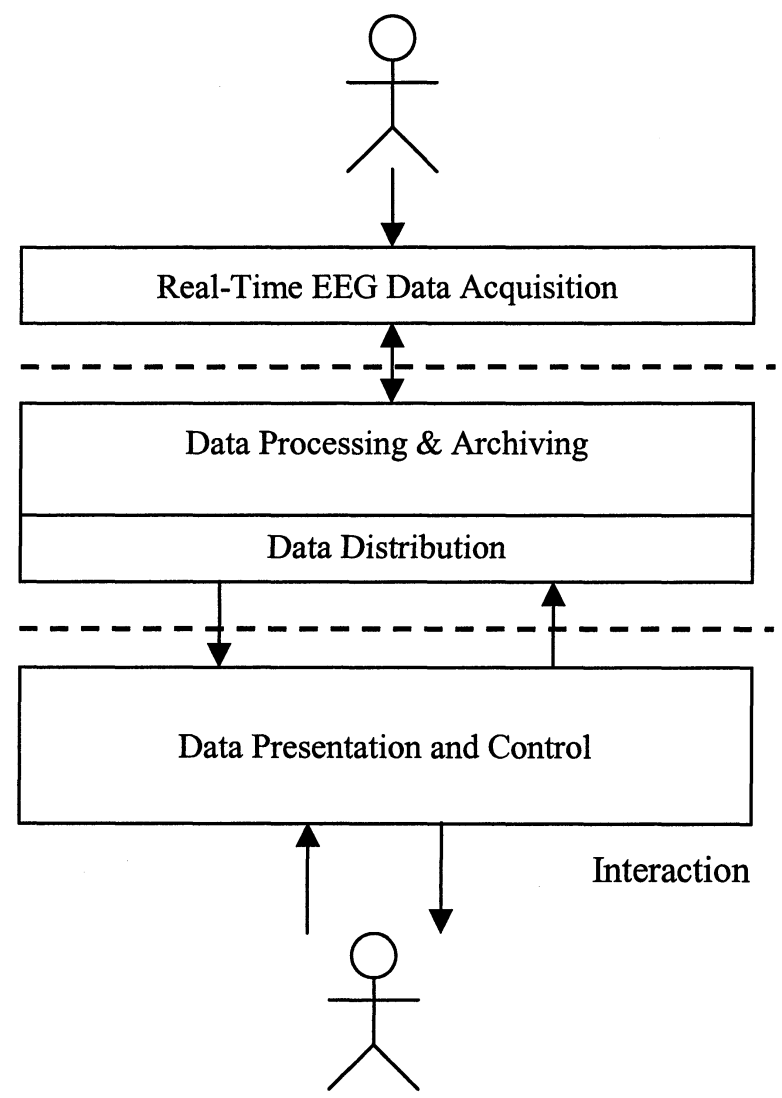

Figure 4. Implementation of the real-time EEG data monitoring and analysisenvironment.

EEG data could be generated either on-line by a data acquisition device or off-line from archive file, and passed to data processing component. Data processing component processes raw EEG data, and prepares data in a form 
suitable for presentation component. Prepared data are placed in the data distribution buffers, and they are distributed on request or pushed through the network. Data presentation and control component presents data, and exports control interface to a user. Data presentation is combination of visual and audio modalities [13].

Having in mind possible infrastructure for Virtual Medical World we have developed three VMD interfaces for data acquisition, data presentation and data distribution: GSM SMS interface, WAP WML interface, and WWW HTML interface.

GSM SMS interfaces handle communication using standard Short Message System (SMS). This interface is used for participants with cellular phones that may not support WAP. The interface allows the following modes of communication:

- Emergency SMS push, which sends SMS message to physicians or medical call center in case of medical emergency;

- SMS query, which allows user to ask for some simple information in medical information systems or in a monitoring system;

- Data distribution SMS push, which periodically sends SMS messages with some monitoring data to physicians.

WAP WML interface manages communication over WAP exchanging messages written in Wireless Markup Language (WML). This interface is used for participants with WAP enabled cellular phones. The interface allows the following modes of communications:

- Emergency WAP push, which sends WML messages to physicians or medical call center in case of medical emergency;

- WML browsing, which allows a participant to browse through information in medical information systems or in monitoring system;

- Data distribution WAP, which periodically sends messages to physicians. These data could be simple text or some 2D graphics with wireless bitmap (WBMP).

WWW HTML interface allows creation of sophisticated client-server applications. Web interface allows use of sophisticated technologies such as Java applets and Virtual Reality Modeling Language (VRML) [12], and supports various communications patterns. Having in mind powerful client hardware in Internet environment, we strongly recommend multimodal presentation as a combination of visual and audio modalities [13]. 


\section{IMPLEMENTATION NOTES}

We implemented several VMDs for real-time EEG monitoring and analysis in Institute for Mental Health in Belgrade. We used MEDELEC 1 A97 EEG machine (MEDILOG BV, Nieuwkoop, The Netherlands) connected to PC-based data acquisition workstation using Data Translation DT2801 A/D converter board. We wrote a custom data acquisition driver written in C. A/D converter module converts 16 channels of EEG in realtime with the sampling frequency of $256 \mathrm{~Hz}$ and 12-bit precision. Raw EEG data are then sent to the processing module of the system. Real time archiving of raw data to PMR is optional. In order to achieve compatibility with existing applications we implemented input filter for off-line data analysis using standard EEG data format generated by RHYTHM 8.0 software (Stellate Systems).

Raw data collected with data acquisition can be presented to the user in real-time or off-line from a file. To allow easy change of processing function, we developed flexible system, in which processing is implemented as a replaceable component with standard input and output. We have implemented several processing components. We implemented power spectrum analysis using the fastest software-processing library in PC environment [14]. Data processing subsystem is implemented in Visual C++ as executable application on Windows NT platform (Figure 5).

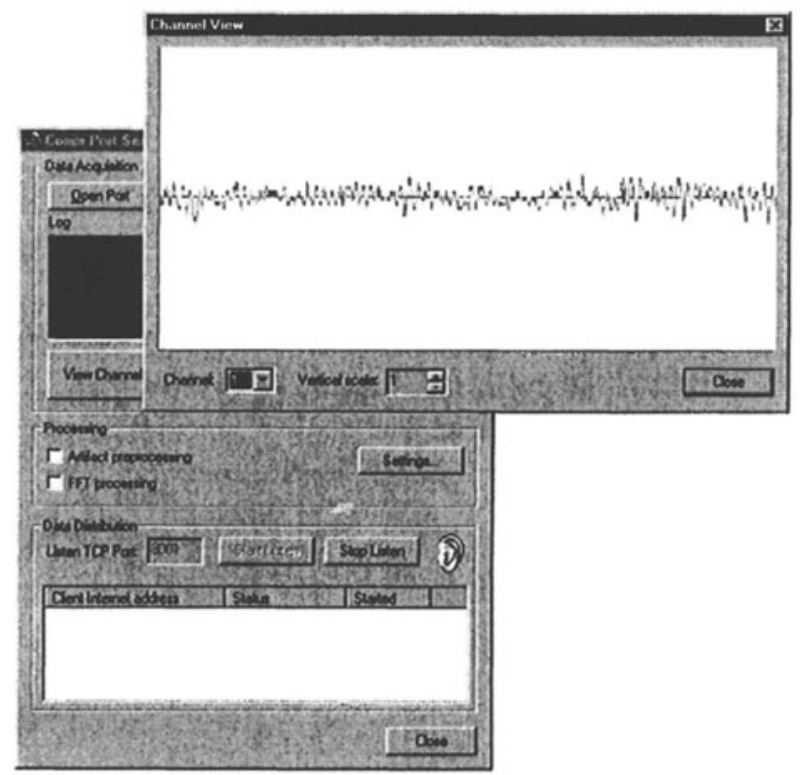

Figure 5. User interface of data acquisition and data processing subsystems. 
Data processing components are also implemented in Visual $\mathrm{C}++$ as separate dynamic link library (DLL) modules used by processing applications. Data distribution is integrated in this application, distributing data to users over TCP connections. We developed simple HTTP server as a part of this solution to distribute HTML and WML pages to clients. We used $\mathrm{XML}$ in our data distribution components as universal format for structured documents and data on the Web.

We developed several VMDs as support for EEG diagnostics. It is possible to use multiple instances of VMDs at the same time as represented in Figure 6.

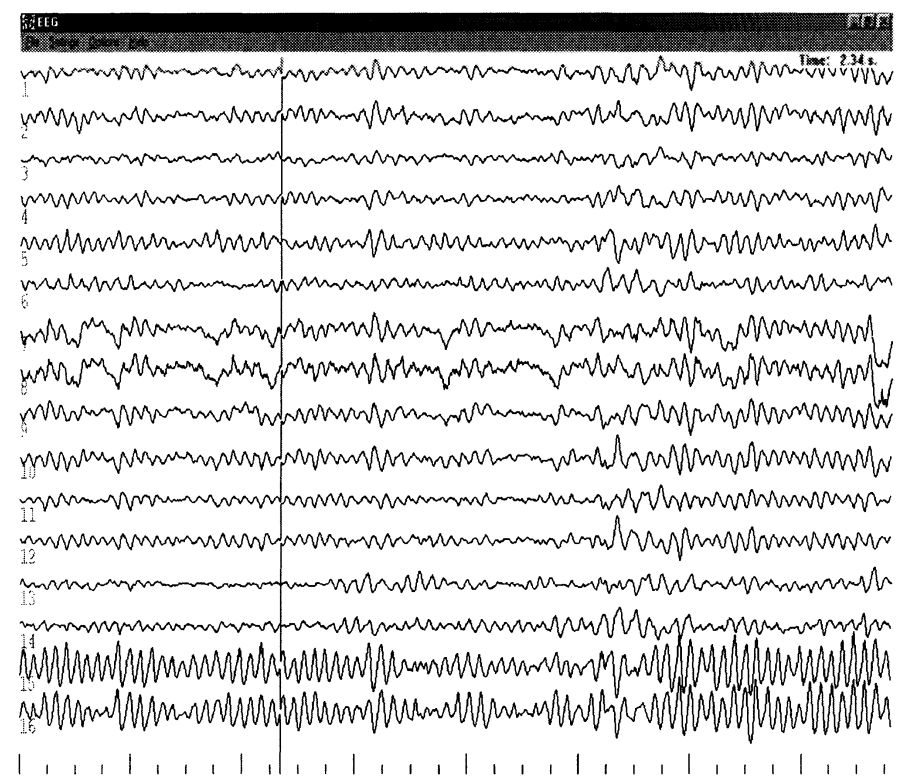

Figure 6. Standard waveform view on EEG signal as default VMD.

Web based VMDs read EEG data using TCP link between control applet and data distribution components. Brain electrical activity is represented with animated topographic maps projected on the 3-D head model. Visualization could be synchronized with sonification of EEG data. Sonification is implemented by modulation of natural sound patterns to reflect certain features of processed data, and create pleasant acoustic environment. This feature is particularly important for prolonged system use. We decided to base our Web based VMDs on Virtual Reality Modeling 
Language (VRML). The VRML is simple language for describing 3-D shapes and interactive environment [12]. VRML is also intended to be universal interchange format for integrated 3-D graphics and multimedia. VRML browsers, as well as authoring tools for the creation of VRML files, are widely available for many different platforms. Today, it is possible to use VRML on small handheld devices, with plugins such as PocketCortona for Windows CE handheld devices [15]. In our system VRML world is controlled by Java applets.

Progress and availability of multimedia and virtual reality (VR) technology made possible perceptual data presentation $[16,17]$. Techniques developed in virtual reality facilitate multiple data stream presentation and navigation through huge data sets. New immersive environments are particularly appropriate to improve insight into complex biomedical phenomena, which are naturally multidimensional. In extension to visualization, which gives predominantly spatial distribution, acoustic rendering may improve temporal cues.

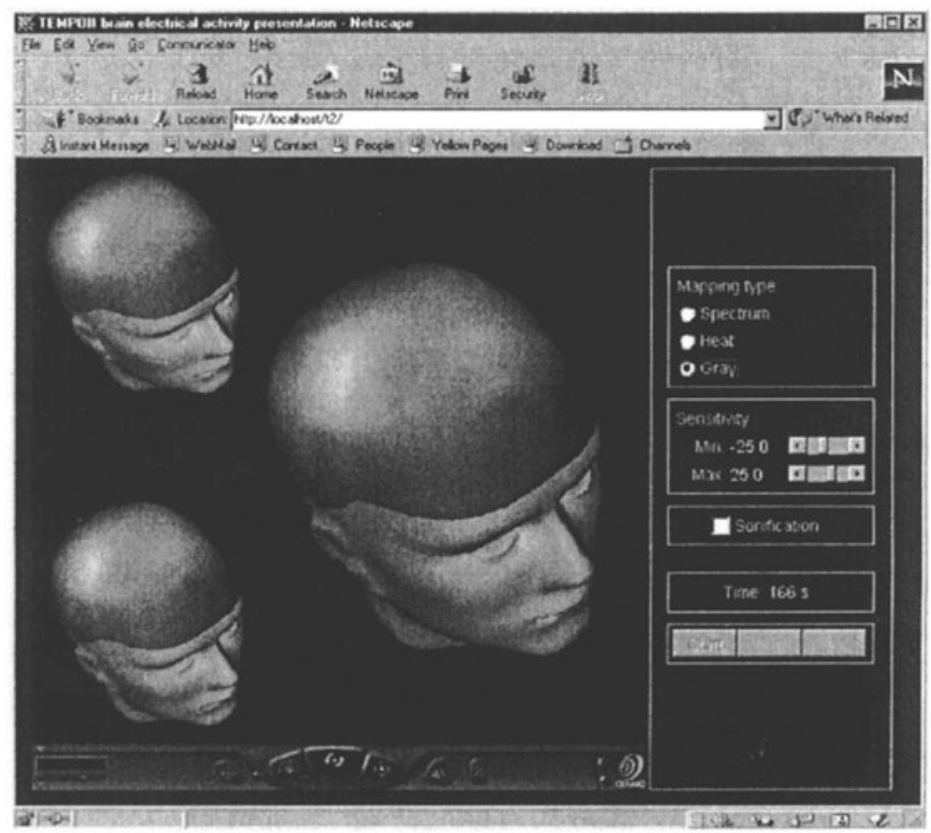

Figure 7. A Web based multimodal VMD for animated topographic map presentation.

In addition to existing 3D presentation with one animated head, we implemented VMD with two small heads that make possible to freeze specific EEG state for comparison with the current state (Figure 7). 
We also developed VMD with speech control to allow user to start and stop presentation of EEG data, and to change mapping type by hands-free speech commands. We have used Microsoft Speech API (SAPI) integrated in control applet. Applet uses services of SAPI via Java Native Interface (JNI) mechanism.

Finally, we developed WAP and SMS based VMDs, for textual and static 2D map presentation using Wireless Bitmap (WBMP) format shown in Figure 8. We tested our WAP VMDs on Nokia 7110 cellular phone, and on number of WAP simulators. WAP devices receive data for presentation from our simple HTTP server via existing WAP gateways. For SMS communication, we used Kannel open source SMS gateway [18]. The SMS interface allows distribution of simple textual messages, and simple queries for medical data.

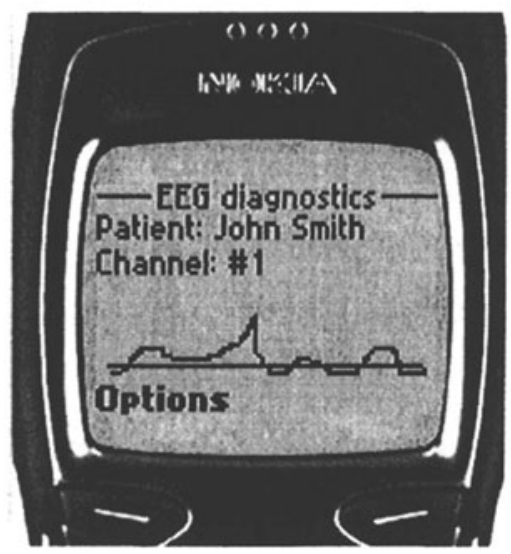

Figure 8. Simple WAP VMD for static 2D map presentation.

\section{EXPERIENCES AND LESSONS LEARNED}

We test our system in Institute for Mental Health in Belgrade. Real-time EEG monitoring and analysis VMDs are still in experimental phase, but we received very good response from physicians.

We were able to experience that new generation of programming environments significantly reduce implementation efforts, providing support for the most frequently used functions. As an example, there is no need to implement viewpoint manipulation in VRML scenes, as it is already supported directly by development tools and VRML viewer. 
Users were satisfied with possibility to choose between various VMDs, and to use more than one VMD at the same time. They liked the possibility to analyze recorded data offline with controlled by conventional multimedia controls. WAP and SMS services could be very useful in case of emergencies.

Our proposed approach is integrated into the prototype of web based medical information system DIMEDAS [19]. During an analysis an appropriate VMD is invoked by simply clicking on the chosen VMD icon associated with the selected recording from local server or a VMD provider (Figure 9).

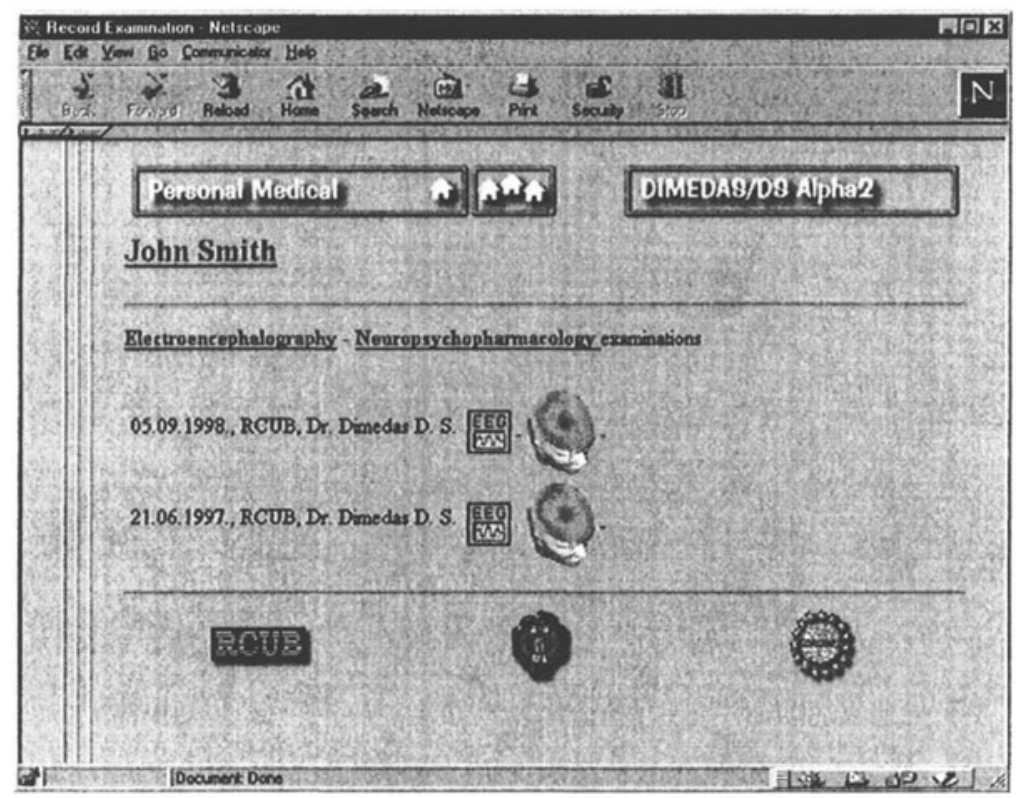

Figure 9. EEG VMDs in DIMEDAS information system; two VMDs are associated with every EEG recording.

\section{CONCLUSION AND FUTURE WORK}

We have presented a telemedical virtual collaborative environment based on the Internet and wireless cellular network technologies. We have applied a novel concept of interactive virtual medical devices (VMDs) to merge remote data acquisition, distributed processing, and data presentation. Through VMD we integrate spatially distributed functions: I/O at patient 
side, distributed data processing, and presentation at physician side. In order to integrate Internet and wireless cellular networks we developed three interfaces for data presentation and data distribution: WWW HTML, WAP WML, and GSM SMS. We used XML in data distribution components as universal format for structured documents on the Web.

We plan to support remote monitoring using wireless personal monitor and cellular phone link connected on request in the case of medical emergencies. As an example, intelligent monitor could recognize epileptic attack analyzing EEG signals, and transfer signal in real time to the physician. Connected to GPS system or other user location services, monitor can inform the paramedics or specialized service about actual position of the patient.

In the current prototype system we didn't implement data encryption. However, information security is crucial for health information system and we are currently working on efficient methods for data encryption and user identification where most problems are not technological. This issue is particularly sensitive in the case of medical emergencies.

\section{REFERENCES}

[1] J.E. Cabral, Y. Kim, "Multimedia Systems for Telemedicine and Their Communication Requirements", IEEE Communications, 34(7):20-27, 1996;

[2] A. Marsh, "EUROMED - Combining WWW and HPCN to suport advanced medical imaging", Int Conf on High Performance Computing and Networking HPCN 1997, Vienna, Austria, 1997;

[3] David J. Goodman, The Wireless Internet: Promises and Challenges, Computer, Vol. 33, No. 7, July 2000;

[4] D.G. Kilman, D.W. Forslund, "An International Collaboratory Based on Virtual Patient Records", Communications of the ACM, 40(8):111-117, 1997;

[5] TeleEEG, A Telemedical Software Package for EEG. University of Calabria, http://www. parcolab.unical.it/Euromed/TeleEEG/

[6] Terry L. Huston, Janis L. Huston, "Is Telemedicine A Practical Reality", Comm. of the ACM, Vol. 43, No. 6, June 2000, pp. 91-95;

[7] Z. Obrenovic, D. Starcevic, E. Jovanov, V. Radivojevic, "An Implementation of Real-time Monitoring and Analysis in Telemedicine", Third IEEE EMBS Information Technology Applications in Biomedicine - Workshop of the International Telemedical Information Society ITAB-ITIS 2000, Arlington, Virginia, November 2000, pp. 74-78;

[8] Neal Leavitt, Will WAP Deliver the Wireless Internet?, Computer, Vol. 33, No. 5: May 2000, pp. 16-20;

[9] E. Jovanov, D. Raskovic, J. Price, A. Moore, J. Chapman, A. Krishnamurthy, "Patient Monitoring Using Personal Area Networks of Wireless Intelligent Sensors," 38th Annual Rocky Mountain Bioengineering Symposium, April 2001, Copper Mountain, Colorado;

[10] E. Jovanov, J. Price, D. Raskovic, K. Kavi, T. Martin, R. Adhami, "Wireless Personal Area Networks in Telemedical Environment", Third IEEE EMBS 
Information Technology Applications in Biomedicine - Workshop of the International Telemedical Information Society ITAB-ITIS 2000, Arlington, Virginia, November 2000, pp. 22-27;

[11] A. Samardzic, E. Jovanov, D. Starcevic, "3D Visualisation of Brain Electrical Activity"; Proceedings of $18^{\text {th }}$ Annual Int'l Conf. IEEE/EMBS, Amsterdam, October 1996;

[12] The Virtual Reality Modeling Language, http://www.vrml.org/Specifications/VRML97

[13] E. Jovanov, D. Starcevic, V. Radivojevic, A. Samardzic, V. Simeunovic, "Perceptualization of biomedical data", IEEE Engineering in Medicine and Biology Magazine, Vol 18, No. 1, pp. 50-55, 1999;

[14] M. Frigo, S. G. Johnson, "FFTW: An Adaptive Software Architecture for the FFT", 1998 ICASSP conference proceedings, Vol. 3, pp.1381-1384, also http://www.fftw.org/

[15] ParallelGraphics Pocket Cortona, $\mathrm{http}: / / \mathrm{www}$.parallelgraphics.com/products/cortonace

[16] E.Jovanov, D. Starcevic, A. Samardzic, A. Marsh, Z. Obrenovic, "EEG analysis in a telemedical virtual world", Future Generation Computer System 15 (1999) 255-263;

[17] W.J. Greenleaf, "Developing the Tools for Practical VR Applications," IEEE EMBS, 15(2), 1996, pp. 23-30;

[18] Kannel - Open Source SMS and WAP Gateway, http://www.kannel.org/

[19] D. Starcevic, E. Jovanov, V. Radivojevic, Z. Obrenovic, A. Samardzic, "Virtual Medical Devices for Telemedical Applications", chapter in Spasic, P., Milosavljevic, I., Jancic-Zguricas, M., Ed's," Telemedicine”, Academy of Medical Sciences of Serbian Medical Association, 2000. 